\title{
A mutual energy formulation for optimal structural design
}

\author{
J.E. Taylor and M.P. Bendsøe
}

\begin{abstract}
This paper presents a variational formulation for the design of elastic structures where the function to be minimized by the optimal design, i.e. the objective, is expressed in abstract form. The resulting statement of necessary conditions is uniformly applicable for all admissible objectives. Both state and adjoint state variables appear directly in the problem statement, and all objectives and the arguments of constraints are scalars. The adjoint pair of state variables appear in symmetric roles via the expression termed "mutual energy". Application of the generalized formulation is demonstrated by treatment of the following examples: design to minimize the maximum value of displacement or to minimize a global measure of stress, design for generalized compliance, design where self-weight is taken into account, and multicriterion design.
\end{abstract}

Key words structural optimization, variational formulations, general objectives, continuum design, mutual energy

1

\section{Introduction}

This paper reports results from an investigation of alternative variational forms for stating problems in structural optimization. In particular, we present a formulation expressed in a form that is general with respect to design objective, i.e. the design objective is stated in abstract

\footnotetext{
Received April 28, 2000

J.E. Taylor ${ }^{1}$ and M.P. Bends $\varnothing \mathrm{e}^{2}$

${ }^{1}$ Department of Aerospace Engineering, University of Michigan, Ann Arbor, MI 48109, USA

e-mail: janos@umich.edu

2 Department of Mathematics, Technical University of Denmark, DK-2800 Lyngby, Denmark

e-mail: bendsoe@mat.dtu.dk
}

form. This is in contrast to the practice that has the specific formulation for minimum compliance design serve most often as the vehicle for presentations on variational developments. With the problem expressed for abstract objective, it becomes possible to consider aspects of analysis, problem type, and method for solution in a general setting, and accordingly to draw conclusions that are relatively more broadly applicable. The work might best be viewed as an experimental engagement in the subject ... judging from our experience, further useful developments are likely forthcoming.

In formulations other than for the classical minimum compliance case, analytical modelling for the coupled analysis and design problem generally involves an associated or adjoint problem. In this case, the significant measure appearing in the optimality condition is a measure symmetric in the primary and associated state variables; this measure has been termed specific mutual energy [applications are discussed by Prager (1974), Dems and Mroz (1995), Rozvany (1989), for example]. In the variational formulation presented here, unit mutual energy appears explicitly as the basis for this new model. The systems governing both primary and adjoint state variables are identified by conventional means, namely as part of the set of necessary conditions for the generalized, coupled analysis and design problem. At the same time, the simplicity of form in the expression for the optimality condition carries over uniformly for the various objectives.

As already noted, for present purposes the objective itself is stated in abstract form, as the integral over the structure of an argument depending generally on structural response and possibly also on design. Necessary conditions include a characterization of the equilibrium problem and an associated equilibrium problem, governing respectively the primary and associated (adjoint) state variables. In this way, the associated problem, which depends on the specified objective, is generated as well in abstract, i.e. general form. Expression of the coupled analysis and design problem in terms of mutual energies has these two boundary value problem statements generated by symmetric operation with respect to each of the primary and associated variable. At the same time, the gen- 
eralized optimality condition has the form where "unit mutual energy" replaces "unit strain energy" of the familiar condition for standard minimum compliance design.

While the concepts which underlie the material of this paper apply to optimal design of structures in general, as it is reported here the development is written for continuum structures. The variational formulation is stated and interpreted in general form, and the reciprocal of this (isoperimetric) problem is presented as well. Application of the generalized formulation is demonstrated by treatment of the following examples: design to minimize the maximum value of displacement or to minimize a global measure of stress, design for generalized compliance, design where self-weight is taken into account, and multicriterion design. Additional details of interpretation, and speculation about possible extensions along the line of this work are considered in the discussion section.

\section{2}

The inner, analyses problem: the primary and adjoint problems

The first development in this excursion into problem form is to consider alternative, variational formulations of analysis problems which as their result will give both response and associated field, often referred to as the adjoint field. The format is thus an analysis setting prepared for design or for design sensitivity analysis.

We consider the elastic deformation $u$ of a continuum structure occupying the domain $\Omega$, and with material properties given through the elasticity tensor $\mathbf{E}_{i j k \ell}$, which is assumed positive definite throughout the domain. Without loss of generality we for simplicity suppose that only (nonzero) body force $f$ is present and that the boundary is fixed, so that $u \in H_{0}^{1}$. Moreover, we consider a design independent objective function $\int_{\Omega} \Phi(u) \mathrm{d} \Omega$, i.e. with no explicit dependence on the design variable (see later for a discussion on design dependent objectives); for the objective function we assume that $\Phi(0)=0, \Phi>0$ and $\Phi^{\prime}(u) \neq 0$ for $u \neq 0$. We now write a variational formulation in terms of an auxiliary scalar variable $Z$ and two fields, the deformation $u \in H_{0}^{1}$ and associated field $\hat{u} \in H_{0}^{1}$ often referred to as the adjoint field,

\section{$\max _{Z, u, \hat{u}} Z$}

subject to

$$
\begin{aligned}
& Z-\int_{\Omega} f \hat{u} \mathrm{~d} \Omega \leq 0, \quad Z-\int_{\Omega} \Phi(u) \mathrm{d} \Omega \leq 0, \\
& \int_{\Omega} \mathbf{E}_{i j k \ell} \varepsilon_{i j}(u) \varepsilon_{k \ell}(\hat{u}) \mathrm{d} \Omega \leq \bar{U} .
\end{aligned}
$$

The load $f$, the form $\Phi$, and $\bar{U}$ are data; $\bar{U}>0$ is a bound on the total mutual energy $\int_{\Omega} \mathbf{E}_{i j k \ell} \varepsilon_{i j}(u) \varepsilon_{k \ell}(\hat{u})$ $\mathrm{d} \Omega$ in $\Omega$, with $\varepsilon(\bullet)$ denoting linearized strains. Note that $(Z, u, \hat{u})=(0,0,0)$ is a feasible point and that at a solution point, the value of $Z$ must be positive. Note as well that the problem (1) is not convex, due to the bilinear form of the mutual energy constraint ${ }^{1}$. However, its construction allows for deriving necessary conditions with a unique solution.

In order to see the link between the formulation above and the standard format of formulating equations for displacements and the adjoint field (cf., e.g. Schechter 1967, Haftka and Gürdal 1992) we derive here the necessary conditions for optimality for the formulation above. From the Lagrangian

$L=-Z+\lambda_{1}\left(Z-\int_{\Omega} f \hat{u} \mathrm{~d} \Omega\right)+\lambda_{2}\left[Z-\int_{\Omega} \Phi(u) \mathrm{d} \Omega\right]+$

$\lambda_{3}\left[\int_{\Omega} \mathbf{E}_{i j k \ell} \varepsilon_{i j}(u) \varepsilon_{k \ell}(\hat{u}) \mathrm{d} \Omega-\bar{U}\right]$,

with Lagrange multiplier $\lambda \geq 0, i=1,2,3$, the necessary conditions are

$\frac{\partial L}{\partial Z}=0: \quad \lambda_{1}+\lambda_{2}=1$,

$\frac{\partial L}{\partial u}=0: \quad \lambda_{3} \int_{\Omega} \mathbf{E}_{i j k \ell} \varepsilon_{i j}(\hat{u}) \varepsilon_{k \ell}(v) \mathrm{d} \Omega=$

$\lambda_{2} \int_{\Omega} \Phi^{\prime}(u) v \mathrm{~d} \Omega$ for all $v$,

$\frac{\partial L}{\partial \hat{u}}=0: \quad \lambda_{3} \int_{\Omega} \mathbf{E}_{i j k \ell} \varepsilon_{i j}(u) \varepsilon_{k \ell}(w) \mathrm{d} \Omega=$

$\lambda_{1} \int_{\Omega} f w \mathrm{~d} \Omega$ for all $w$.

Under the condition that all multipliers are nonzero [i.e. all bounds in (1) are active], (5) is, up to a scaling, the equilibrium equation for the displacement, while equation (4) is the so-called adjoint equation for the associated field $\hat{u}$.

In order to see that all multipliers are nonzero, note that also the switching conditions

$\lambda_{1}\left(Z-\int_{\Omega} f \hat{u} \mathrm{~d} \Omega\right)=0$,

1 In general the objective function $\Phi$ can depend on $u$ and its gradient as well as higher order derivatives. In such a case the symbol $\Phi^{\prime}$ requires suitable interpretaton. For simplicity here we just write $\Phi$ generically as $\Phi(u)$ 
$\lambda_{2}\left[Z-\int_{\Omega} \Phi(u) \mathrm{d} \Omega\right]=0$,

$\lambda_{3}\left[\int_{\Omega} \mathbf{E}_{i j k \ell} \varepsilon_{i j}(u) \varepsilon_{k \ell}(\hat{u}) \mathrm{d} \Omega-\bar{U}\right]=0$

are required to hold. First, we note that if $\lambda_{3}=0$, then (5) implies that $\lambda_{1}=0$ (as $f$ is nonzero), so from (3) we have $\lambda_{2}=1$. Finally, $\lambda_{2}=1, \lambda_{3}=0$ gives a contradiction, as this will require that $\Phi^{\prime}(u)=0$, i.e. either a contradiction to our assumptions, or $u=0$ and $Z=0$, which is not the maximum [in this case $(Z, u, \hat{u})=(0,0, \hat{u})$ with $\int_{\Omega} f \tilde{u}$ $\mathrm{d} \Omega=0$ are stationary, nonoptimal points].

The above thus shows that at the optimum, $\lambda_{3}>0$ and [via (8)] the constraint on mutual energy is active. Moreover, we can from (4) and (5) then conclude that also $\lambda_{1}, \lambda_{2}>0$, as a zero value would give a zero displacement or associated field, in contradiction with $\int_{\Omega} \mathbf{E}_{i j k \ell} \varepsilon_{i j}(u) \varepsilon_{k \ell}(\hat{u}) \mathrm{d} \Omega=\bar{U}>0$. Thus the lower bounds in (1) given by $Z$ are also active at the optimum.

With $\lambda_{1}, \lambda_{2}, \lambda_{3}>0,(4)$ and (5) thus uniquely determines the fields $u, \hat{u}$, and the remaining equations (3) and (6)-(8) gives four equations to determine the values of these multipliers and the optimal bound $Z$.

This analysis shows that we can give a variational form covering at once the primary analysis and the associated adjoint problems. From a computational point of view one can take the necessary conditions as the basis for such developments (these conditions are unique), in which case one will in reality use the standard approach of solving first for the displacements and then for the adjoint field, using the factorization of the stiffness matrix used for the primal problem to simplify computations for the associated problem (simple back-substitution). Alternatively, one can use iterative techniques to solve (1) as stated, in which case the computational procedure can be similar to iterative solvers for the equilibrium problem. The possibility that such an approach might lead to new improved computational schemes is left open for speculation.

For completeness let us here remind the reader that for a design problem with respect to a scalar design variable $D$, written in (abstract) form as

$\min _{D} F(D)=\int_{\Omega} \Phi[u(D)] \mathrm{d} \Omega$,

where $u(D)$ solves

$$
\int_{\Omega} \mathbf{E}_{i j k \ell}(D) \varepsilon_{i j}(u) \varepsilon_{k \ell}(v) \mathrm{d} \Omega=\int_{\Omega} f v \mathrm{~d} \Omega \quad \text { for all } v,
$$

the derivative (the design sensitivity) of the functional $F$ can be found from the equations (see, e.g. Haftka and
Gürdal 1992),

$F^{\prime}(D)=-\int_{\Omega} E_{i j k \ell}^{\prime}(D) \varepsilon_{i j}(u) \varepsilon_{k \ell}(\hat{u}) \mathrm{d} \Omega$,

where $\hat{u}$ solves

$\int_{\Omega} \mathbf{E}_{i j k \ell}(D) \varepsilon_{i j}(\hat{u}) \varepsilon_{k \ell}(v) \mathrm{d} \Omega=\int_{\Omega} \Phi^{\prime}[u(D)] v \mathrm{~d} \Omega$,

for all $v$.

The last equation is what is normally labelled as the adjoint equation for the adjoint field $\hat{u}$ (for a nonsymmetric operator this would involve the adjoint operator).

Here we note that our analysis of problem (1) has shown that at the solution point all constraints are active and the variable $u$ is the displacement (up to a scaling, dependent on the choice of $\bar{U}$ ). Thus the value of the variable $Z$ at the solution point is equal to the objective function and the design problem written above can alternatively be stated as

$$
\begin{array}{lc}
\min _{D} & \max \\
Z, u, \hat{u} \\
& \text { subject to: } \\
Z-\int_{\Omega} f \hat{u} \mathrm{~d} \Omega \leq 0 \\
Z-\int_{\Omega} \Phi(u) \mathrm{d} \Omega \leq 0 \\
\\
\int_{\Omega} \mathbf{E}_{i j k \ell}(D) \varepsilon_{i j}(u) \varepsilon_{k \ell}(\hat{u}) \mathrm{d} \Omega \leq \bar{U}
\end{array}
$$

Below we will rewrite this in a somewhat more convenient fashion, using an interchange of objective and the mutual energy constraint.

Before proceeding to statements for more general cases we close this introductory presentation by considering the very well-known case of minimum compliance. Here problem (1) takes the form

$\max _{Z, u, \hat{u}} Z$

subject to

$$
\begin{aligned}
& Z-\int_{\Omega} f \hat{u} \mathrm{~d} \Omega \leq 0, \quad Z-\int_{\Omega} f u \mathrm{~d} \Omega \leq 0, \\
& \int_{\Omega} \mathbf{E}_{i j k \ell} \varepsilon_{i j}(u) \varepsilon_{k \ell}(\hat{u}), \mathrm{d} \Omega \leq \bar{U},
\end{aligned}
$$

where we have symmetry with respect to the two fields $u, \hat{u}$ (i.e. interchanging these variables results in the same problem). The uniqueness indicated above for the general case thus directly shows that these two fields must be equal. The result is, of course, well-known (cf., e.g. Prager and Taylor 1968, Taylor 1987). Still it is noteworthy that the minimum compliance design problem is identified as an example within the general development directly from the structure of (9) and without need of further analysis. 
For illustration of some possible generalizations of the formulation presented above, we first treat a case where we consider multiple objectives under one load and propose to employ a min-max format to cope with the multiple objectives. For the analysis (primary and associated problems) we write:

$\max _{Z, u, \hat{u}} Z$

subject to

$Z-\int_{\Omega} f \hat{u} \mathrm{~d} \Omega \leq 0, \quad Z-\int_{\Omega} \Phi_{1}(u) \mathrm{d} \Omega \leq 0$,

$Z-\int_{\Omega} \Phi_{2}(u) \mathrm{d} \Omega \leq 0$

$\int_{\Omega} \mathbf{E}_{i j k \ell} \varepsilon_{i j}(u) \varepsilon_{k \ell}(\hat{u}) \mathrm{d} \Omega \leq \bar{U}$.

By an analysis similar to the one above, the adjoint problem is expressed in weak form as

$\int_{\Omega} \mathbf{E}_{i j k \ell} \varepsilon_{i j}(\hat{u}) \varepsilon_{k \ell}(v) \mathrm{d} \Omega=\gamma_{1} \int_{\Omega} \Phi_{1}^{\prime}(u) v \mathrm{~d} \Omega+$

$\gamma_{2} \int_{\Omega} \Phi_{2}^{\prime}(u) v \mathrm{~d} \Omega \quad$ for all $v$

where the nonnegative parameters $\gamma_{1}, \gamma_{2}$ are nonzero only if the corresponding objective bound is active. This corresponds to the spanning of the generalized gradient for a nonsmooth design problem which seeks to minimize

$\tilde{F}(D)=\max \left\{\int_{\Omega} \Phi_{1}[u(D)] \mathrm{d} \Omega, \int_{\Omega} \Phi_{2}[u(D)] \mathrm{d} \Omega\right\}$,

where $u(D)$ solves

$$
\begin{aligned}
& \int_{\Omega} \mathbf{E}_{i j k \ell}(D) \varepsilon_{i j}(u) \varepsilon_{k \ell}(v) \mathrm{d} \Omega= \\
& \int_{\Omega} f v \mathrm{~d} \Omega \text { for all } v .
\end{aligned}
$$

3

\section{A reciprocal form in mutual energy}

The analysis problem (1) appears in a form where the design dependent mutual energy is part of the constraints. For a number of cases it is convenient to use an equivalent (reciprocal) form, where the mutual energy becomes the objective function and where a positive lower bound on the variable $Z$ is imposed. The variable $Z$ is replaced by $\Xi$ to make a distinction in the role of the variable. Then, the equivalent form is stated as

$\min _{\Xi, u, \hat{u}} \int_{\Omega} \mathbf{E}_{i j k \ell} \varepsilon_{i j}(u) \varepsilon_{k \ell}(\hat{u}) \mathrm{d} \Omega$

subject to

$\Xi-\int_{\Omega} f \hat{u} \mathrm{~d} \Omega \leq 0, \quad \Xi-\int_{\Omega} \Phi(u) \mathrm{d} \Omega \leq 0$,

$\Xi-\Xi \leq 0$

with a lower bound $\Xi>0$. It is here straightforward to check that the necessary conditions for this problem also results in the equilibrium problem and the adjoint problem associated with the functional $\int_{\Omega} \Phi(u) \mathrm{D} \Omega$. The uniqueness of the solutions to these equations imply the complete equivalence, and one obtains that for any choice of upper bound $\bar{U}$ for (1) and corresponding optimal value $Z^{*}$, the solution to (10) with lower bound $\underline{\Xi}=Z^{*}$ results in an optimal value of the mutual energy equal to $\bar{U}$, and vice versa. Also the displacement field and the adjoint field coincide.

Here the reciprocal form is used to consider the case of a multiple purpose, multiple load design problem. For this, we should formulate multiple equilibrium problems and a number of associated adjoint problems arising from a set of functionals. Thus with $M$ loads and associated functionals we can write the problem

$\max _{Z_{m}, u_{m}, \hat{u}_{m}} \sum_{m=1}^{M} Z_{m}$

subject to

$Z_{m}-\int_{\Omega} f_{m} \hat{u}_{m} \mathrm{~d} \Omega \leq 0, \quad Z_{m}-\int_{\Omega} \Phi_{m}\left(u_{m}\right) \mathrm{d} \Omega \leq 0$,

$\int_{\Omega} \mathbf{E}_{i j k \ell} \varepsilon_{i j}\left(u_{m}\right) \varepsilon_{k \ell}\left(\hat{u}_{m}\right) \mathrm{d} \Omega \leq \bar{U}_{m}$,

$m=1, \ldots, M$,

where the optimal value will be equal to a sum of the functionals $\int_{\Omega} \Phi_{m}\left(u_{m}\right) \mathrm{d} \Omega$ at equilibrium. Note that each set of constraints is mutually independent, so that (11) in practise is just a collection of independent problems of the form (1). This also means that the reciprocal form of (11) has the form

$\min _{\Xi_{m}, u_{m}, \hat{u}_{m}} \sum_{m=1}^{M} \int_{\Omega} \mathbf{E}_{i j k \ell} \varepsilon_{i j}\left(u_{m}\right) \varepsilon_{k \ell}\left(\hat{u}_{m}\right) \mathrm{d} \Omega$

subject to 


$$
\begin{aligned}
& \Xi_{m}-\int_{\Omega} f_{m} \hat{u}_{m} \mathrm{~d} \Omega \leq 0, \quad \Xi_{m}-\int_{\Omega} \Phi_{m}\left(u_{m}\right) \mathrm{d} \Omega \leq 0, \\
& \Xi_{m}-\Xi_{m} \leq 0, \quad m=1, \ldots, M .
\end{aligned}
$$

\section{4}

\section{The mutual energy formulation as a basis for design problems}

We continue here using the reciprocal forms of the analysis problems above as the basis for formulating design problems which treat design objectives defined in terms of functionals $\int_{\Omega} \Phi_{m}\left(u_{m}\right)$. Thus according to the mutual energy format above, the design problem seeks the maximal mutual energy for a lower bound $\Xi$ on performance level. Taking in abstract terms the elasticity tensor as the design variable we can write this problem as

$\max _{E} \min _{\Xi, u, \hat{u}} \int_{\Omega} \mathbf{E}_{i j k \ell} \varepsilon_{i j}(u) \varepsilon_{k \ell}(\hat{u}) \mathrm{d} \Omega$

subject to

$\Xi-\int_{\Omega} f \hat{u} \mathrm{~d} \Omega \leq 0, \quad \Xi-\int_{\Omega} \Phi(u) \mathrm{d} \Omega \leq 0$,

$\Xi-\Xi \leq 0, \quad E \in \mathbf{E}_{\text {ad }}$,

where $\mathbf{E}_{\mathrm{ad}}$ denotes the set of admissible tensors. As an example we can consider the design problem with an unrestricted material tensor, and use the Frobenius norm as a cost measure (cf. Bendsøe et al. 1994, 1995)

$\max _{E \geq 0} \min _{\Xi, u, \hat{u}} \int_{\Omega} \mathbf{E}_{i j k \ell} \varepsilon_{i j}(u) \varepsilon_{k \ell}(\hat{u}) \mathrm{d} \Omega$

subject to

$\Xi-\int_{\Omega} f \hat{u} \mathrm{~d} \Omega \leq 0, \quad \Xi-\int_{\Omega} \Phi(u) \mathrm{d} \Omega \leq 0$,

$\Xi-\Xi \leq 0$

$\int_{\Omega} \Psi(E) \mathrm{d} \Omega \leq V, \quad \Psi(E)=\left(\mathbf{E}_{i j k \ell} \mathbf{E}_{i j k \ell}\right)^{1 / 2}$,

$0 \leq \Psi_{\min } \leq \Psi(E) \leq \Psi_{\max }$,

where $E \geq 0$ signifies that $E$ is a positive semi-definite tensor.

In this max-min problem, standard convexity based saddle-point theorems cannot be applied. However, it is still useful to consider an interchange of the maximization and minimization which here with the design independent functional $\int_{\Omega} \Phi(u) \mathrm{d} \Omega$ will lead to an associated pointwise problem of the form

$$
\begin{gathered}
\max _{E \geq 0} \quad \mathbf{E}_{i j k \ell} \varepsilon_{i j}(u) \varepsilon_{k \ell}(\hat{u}) \\
\left(\mathbf{E}_{i j k \ell} \mathbf{E}_{i j k \ell}\right)^{1 / 2} \leq \rho
\end{gathered},
$$

where $\rho$ denotes the local assignment of resource, as measured by (c.f. Bendsøe et al. 1994). A solution to this associated problem will constitute an expression of what local material properties will be optimal for the problem at hand. As studied in detail in the recent book by Cherkaev (2000), a similar problem appears when studying structures made from mixtures of material and void, and we refer to this reference for a broad treatment of this type of problem format.

\section{5}

\section{Examples}

In the following we show the problem format for a few example design settings, using for simplicity a 1-D formulation.

\section{1}

\section{Example 1. Generalized compliance}

Assume that the goal of the design process is to limit a global measure of displacement expressed as $\int_{\Omega} q|u| \mathrm{d} x$ with a given weighting function q that may vary over the domain.

For the general format above to handle the numerical value of this functional, one can introduce an auxiliary function $\beta(x)$ in the problem statement and have a suitable formulation written as

$\max _{D} \min _{\Xi, u, \hat{u}} \int_{\Omega} D u^{\prime} \hat{u}^{\prime} \mathrm{d} x$

subject to

$\Xi-\int_{\Omega} f \hat{u} \mathrm{~d} x \leq 0, \quad \Xi-\int_{\Omega} q \beta \mathrm{d} x \leq 0$,

$u(x)-\beta(x) \leq 0, \quad-u(x)-\beta(x) \leq 0$,

$\underline{\underline{E}}-\Xi \leq 0, \quad D \in \Delta_{\mathrm{ad}}$.

Here the design variable $D$ represents structural stiffness $E A, f$ symbolizes applied load, and as above, the displacement and adjoint fields are denoted by $u$ and $\hat{u}$, respectively. The load $f$, the weighting function $q$, and the lower bound $\Xi$ are given data for the problem. 
5.2

\section{Example 2. Global measure of streses}

Here we treat a stress measure written as $\int_{\Omega} w\left|E u^{\prime}\right|^{2} \mathrm{~d} x$ with a given weighting function $w$ that may vary over the domain. This is now a design dependent objective. In this case the analysis and associated problem for the adjoint can still be handled via the general format of (1), but care has to be taken when considering necessary condition and gradient information for a design problem involving such a design dependent functional. Here we write an example design problem as

$\max _{E} \min _{\Xi, u, \hat{u}} \int_{\Omega} E A u^{\prime} \hat{u}^{\prime} \mathrm{d} x$

subject to

$\Xi-\int_{\Omega} f \hat{u} \mathrm{~d} x \leq 0, \quad \Xi-\int_{\Omega} w\left(E u^{\prime}\right)^{2} \mathrm{~d} x \leq 0$,

$\Xi-\Xi \leq 0, \quad E \in \mathbf{E}_{\mathrm{ad}}$.

To obtain necessary conditions for optimality with respect to the design variable $E$ one can consider the problem written as

$\max _{E \in \mathbf{E}_{\mathrm{ad}}} \min _{\Xi, u, \hat{u}}\left\{\int_{\Omega} E A u^{\prime} \hat{u}^{\prime} \mathrm{d} x \mid \Xi-\int_{\Omega} f \hat{u} \mathrm{~d} x \leq 0\right.$,

$\left.\Xi-\int_{\Omega} w\left(E u^{\prime}\right)^{2} \mathrm{~d} x \leq 0, \Xi-\Xi \leq 0\right\}$,

where the derivative of the inner functional $F(E)$ can be expressed as

$F^{\prime}=u^{\prime} \hat{u}^{\prime}-2 \lambda_{2} w E\left|u^{\prime}\right|$.

Here $\lambda_{2}$ is the Lagrange multiplier for the stress constraint in the inner problem, evaluated at the solution point.

\section{6}

\section{Variations of the theme}

This last section will describe two situations where some slight variation of the general format is required to handle the type of criterion. First we consider a case where the objective is to minimize the maximum displacement under a given load, i.e. the functional under study is $\max _{x \in \Omega}|u(x)|$. In this case we can use a bound formulation (cf. Taylor 1987), so that the objective function is actually a scalar parameter, here named $\beta$, which controls the values of the displacement throughout the structure. A suitable formulation is then

$\max _{D} \min _{\Xi, u, \hat{u}} \int_{\Omega} D u^{\prime} \hat{u}^{\prime} \mathrm{d} x$ subject to

$\Xi-\int_{\Omega} f \hat{u} \mathrm{~d} x \leq 0, \quad \Xi-\beta \leq 0$,

$u(x)-\beta(x) \leq 0, \quad-u(x)-\beta \leq 0$,

$\Xi-\Xi \leq 0, \quad D \in \Delta_{\mathrm{ad}}$.

The last example is concerned with treating problems with self-weight, i.e. a problem where a design dependent load affects the design. Here we take the objective to be the work done by the dead-load, for a displacement given by the live load only, giving a formulation

$\max _{D} \min _{\Xi, u, \hat{u}} \int_{\Omega} D u^{\prime} \hat{u}^{\prime} \mathrm{d} x$

subject to

$\Xi-\int_{\Omega} f \hat{u} \mathrm{~d} x \leq 0, \quad \Xi-r \int_{\Omega} D \gamma u \mathrm{~d} x \leq 0$,

$\Xi-\Xi \leq 0, \quad D \in \Delta_{\mathrm{ad}}$.

The self-weight term corresponds to the case where "weight" is given by $\gamma$, i.e. unit weight is proportional (by $\gamma$ ) to the design $D$. The coeffcient $r$ is used to enter the importance of "dead load" relative to live load. In this form of problem statement total displacement is given by $u+\hat{u}$.

\section{7}

\section{Discussion}

As was pointed out in Sect. 2, a minmax formulation for the usual minimum compliance problem is imbedded within the present mutual energy formulation, and this is easily recognized through the symmetry in the problem statement. Mutual strain energy reduces to strain energy for the latter problem, and the two performance constraints of the general problem collapse to one. Also, considering the modification to cover multipurpose design, this extension is achieved as simply for the present generalized objective formulation as it is for the classical minimum compliance case.

We note that our concern in this investigation centers on the handling of the analysis problem for general objective functions in a formulation that is compact and convenient for design optimization. The important considerations related to design for arbitrary material properties and generalizations of the cost constraints are beyond the scope of this paper; we refer the reader to the discussions on these aspects of problem formulation in for example Bendsøe (1995), Rodrigues et al. (1999), Taylor (1998, $2000)$, and in references quoted in these publications. 
Along a different line, it might be argued that the trend in our subject over the recent decade has relatively more attention paid to development of numerical analysis and means for computational treatment than to fundamental modelling. Considering the significance overall of having established models available - their use as a basis to devise and confirm schemes for computational solution, their usefulness toward gaining general insight and understanding about the effects on behaviour of efforts to improve design, the convenience of the "top-down" quality characteristic of variational treatment, namely that it provides a unifying basis for studies where the object is to enlarge on what is covered in the modelling, and so on - perhaps it is reasonable that the role of research in the fundamental format of modelling should be viewed with renewed interest. Certainly the developments reported here comprise no more than a brief excursion into the area.

Acknowledgements Support provided by the Ford Motor Company (JT) and the Villum Kann-Rasmussen Foundation (MPB) is gratefully aknowledged.

\section{References}

Bendsøe, M.P. 1995: Optimization of structural topology, shape and material. Berlin, Heidelberg, New York: Springer

Bendsøe M.P.; Díaz, A.R.; Lipton, R.; Taylor, J.E. 1995: Optimal design of material properties and material distribution for multiple loading conditions. Int. J. Num. Meth. Engng. 38, $1149-1170$

Bendsøe, M.P.; Guedes, J.M.; Haber, R.B.; Pedersen, P.; Taylor, J.E. 1994: An analytical model to predict optimal ma- terial properties in the context of optimal structural design. J. Appl. Mech. 61, 930-937

Cherkaev, A. (2000): Variational methods for structural optimization. Berlin, Heidelberg, New York: Springer

Dems, K.; Mroz, Z. 1995: Shape sensitivity in mixed DirichletNeumann boundary value problems. Eur. J. Mechs./A Solids 14, 169-203

Haftka, R.T.; Gürdal, Z. 1992: Elements of structural optimization. Dordrecht: Kluwer

Prager, W. 1974: Introduction to structural optimization. CISM Courses \& Lectures No. 212. Vienna: Springer

Prager, W.; Taylor, J.E. 1968: Problems of optimal structural design. J. Appl. Mech. 35, 102-106

Rodrigues, H.C.; Soto, C.; Taylor, J.E.; 1999: A model to predict efficient two-component composite structures. Struct. Optim. 17, 186-198

Rozvany, G.I.N. 1989: Structural design via optimality criteria. Dordrecht: Kluwer

Schechter, R.S. 1967: The variational method in engineering. New York: McGraw-Hill

Taylor, J.E. 1987: Distributed parameter optimal structural design: some basic problem formulations and their applications. In: Mota Soares, C.A. (ed.) Computer aided optimal design: structural and mechanical systems, pp.3-85. Berlin, Heidelberg, New York: Springer

Taylor, J.E. 1998: An energy model for the optimal design of linear continuum structures. Struct. Optim. 16, 116-127

Taylor, J.E. 2000: Addendum to: An energy model for the optimal design of continuum structures. Struct. Multidisc. Optim. 19, 317-320 